\title{
ASSESSING THE IMPACT OF A NUTRITION-FOCUSED QUALITY IMPROVEMENT PROGRAM IN CARDIOPULMONARY PATIENTS
}

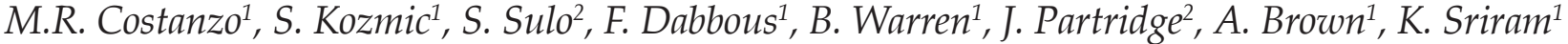

\begin{abstract}
Background: Patients with cardiopulmonary diagnoses are at high risk for hospital readmissions and prolonged hospitalizations. Nutrition-focused quality improvement programs (QIPs) can improve the care of malnourished hospitalized patients. Objectives: Data collected previously was analyzed to evaluate the impact of a nutrition-focused QIP on health outcomes in patients with cardiopulmonary diseases. Design: The QIP consisted of malnutrition risk screening, prompt initiation of oral nutritional supplements (ONS), and nutrition education. Setting: A total of 4 hospitals implemented the QIP-2 teaching hospitals and 2 community hospitals. Participants: Eligible QIP participants were hospitalized patients with any diagnosis, 18 years of age or older, at risk for malnutrition at admission, and able to consume food and beverages orally. Measurements: Data collected from the QIP patients was compared to data from historical controls to assess differences in readmission rates and length of stay (LOS). Results: Patients were mainly older adults (66 \pm 17.4 years) and non-obese (85\%). Univariate analysis showed significant reductions in 30-day readmission rates for the QIP group when compared with the controls (13.9\% for QIP vs. $26.4 \%$ for controls), with the QIP group experiencing a 55\% reduction in the odds of being readmitted (OR $=0.45, \mathrm{p}=0.006)$. Similarly, a significant reduction in LOS was reported for the QIP group (5.4 \pm 5.7 days for QIP vs. $6.8 \pm 5.7$ days for controls) corresponding to a relative risk reduction $(\mathrm{RR})$ of $20 \%(\mathrm{RR}=0.80, \mathrm{p}=0.0085)$. Logistic regression adjusting for patient characteristics showed that the QIP patients were $33 \%$ less likely to be readmitted $(p=0.33)$, and had a $6 \% R R(R R=0.94, p=0.55)$ in LOS versus controls. Conclusions: Malnourished hospitalized cardiopulmonary patients participating in a nutrition-focused QIP experienced fewer readmissions and improved LOS compared to controls. These results underscore the importance of nutrition-focused interventions as a key part of treatment for cardiopulmonary patients.
\end{abstract}

Key words: Quality improvement, malnutrition, cardiopulmonary, readmission rates, length of stay.

\section{Introduction}

Cardiopulmonary patients are at high risk for hospital readmissions and prolonged hospitalizations. About $25 \%$ of patients hospitalized with heart failure (HF) are readmitted within 30 days (1-4). Efforts to reduce readmissions after HF hospitalizations have been largely unsuccessful; according to national data, readmission rates for these patients have not dropped significantly during the past two decades (5). Advancements in treatment have improved in-hospital survival for cardiopulmonary patients, resulting in a greater number of survivors being discharged into the community and thus at risk for readmission (6). The hospital length of stay (LOS) for cardiopulmonary patients also remains high at an average of 6.33 days compared to 4.5 days for all patients (7). Risk factors such as advanced age

1. Advocate Health Care, Downers Grove, Illinois, USA; 2. Abbott Nutrition Research \& Development, Columbus, Ohio, USA; All authors take responsibility for all aspects of the reliability and freedom from bias of the data presented and their discussed interpretation.

Corresponding Author: Maria Rosa Costanzo, MD, 801 South Washington Street, Naperville, Illinois 60540, Email: mariarosa.costanzo@advocatehealth.com and malnourishment may further extend a patient's LOS. Studies have shown that the LOS for malnourished patients is double that of their well-nourished counterparts (8), and older patients hospitalized for cardiopulmonary diseases could experience LOS exceeding 12 days (9). In fact, the negative consequences of malnutrition on health outcomes are even more pronounced among older patient populations (65 years and older) who are at higher risk for developing malnutrition as a result of limited dietary intake and diet quality and multiple chronic conditions $(10,11)$.

Bradley et al. outlined six strategies that hospitals in the United States commonly use to reduce 30-day readmission rates among HF patients (i.e., partnering with community physicians and physician groups, partnering with local hospitals, having nurses responsible for medication reconciliation, arranging for follow-up visits before discharge, having a process in place to send all discharge or electronic summaries directly to the patient's primary care physician, and assigning staff to follow up on test results after the patient is discharged). However, none of these strategies included nutrition risk assessment and treatment (12), a key omission 
as malnutrition increases morbidity and mortality, specifically in catabolic state diseases such as HF (13). Malnutrition affects about $50 \%$ of patients worldwide upon admission to the hospital $(14,15)$ and many patients also experience a decline in nutritional status during their hospital stay, leading to higher 30-day readmission rates and prolonged hospitalizations. As a result of this increased healthcare resource utilization, the annual economic burden of disease-associated malnutrition has been found to be approximately $\$ 157$ billion (in 2010 USD). For chronic obstructive pulmonary disease (COPD) the economic burden is $\$ 43.2$ billion, while for coronary heart disease the economic burden is estimated to be $\$ 23.9$ billion (15).

Interventions that decrease hospital readmissions are crucial to improve the quality and lower the cost of care for patients with cardiopulmonary diagnoses, especially the older patient populations. Quality improvement programs (QIP) are an example of these types of interventions that can dramatically improve patient outcomes. A study by Nuckols et al. found that QIPs can successfully improve health outcomes for patients with HF, with an average readmission reduction of $12.1 \%$ and a mean net savings to the health system per patient of $\$ 972$ (16). Nutrition is one area where QIPs can make an impact on patient outcomes. Patients with cardiopulmonary diagnoses often have a number of chronic comorbid conditions, notably diabetes, which complicate their nutrition status and lead to poorer health outcomes (17).

Several studies have found that addressing the nutritional needs of hospitalized patients through QIPs reduces both readmission and LOS (18-19). Therefore, the purpose of this post-hoc analysis was to evaluate the impact of nutrition-focused QIP interventions on 30-day unplanned readmissions and LOS in the cohort of patients with cardiopulmonary diagnoses.

\section{Methods}

Eligible QIP participants were hospitalized patients with any diagnosis, 18 years of age or older, at risk for malnutrition (malnutrition scoring tool [MST] score $\geq 2$, see Appendix A) at admission, and able to consume food and beverages orally.

The QIP consisted of malnutrition risk screening at admission via electronic medical record (EMR), prompt initiation of oral nutritional supplements (ONS) for at-risk patients, and nutrition education for the inpatients and caregivers. All QIP patients were screened at hospital admission using the MST (20). The EMR was upgraded to trigger appropriate dietitian consultations and selection of standard or disease-specific ONS for all at-risk patients by the admitting nurse as long as there was no physician order to keep the patient nil by mouth. ONS was either ordered by EMR-cue based on MST score or manually by the dietitian. The treatment protocol provided two bottles of ONS daily, delivered with meals. Patients were matched to the ONS formula type per the patient's overall dietary orders prescribed by the admitting physician (i.e., standard, diabetes-specific, or renal-specific ONS). This study was approved by the Advocate Health Care (AHC) Institutional Review Board. The study is registered with clinicaltrials.gov (NCT02262429). Additional details regarding the study methodology were described by Sriram et al (18).

Data were divided into categories based on diagnosis related groups (DRGs). The cardiopulmonary DRG was the largest group and was comprised of 310 patients. Common cardiopulmonary diagnoses using the International Statistical Classification of Diseases and Related Health Problems - 9th edition (ICD-9) codes identified included HF (ICD 428-428.99), hypertension (ICD 401-401.9, I10, 437.2, 642-642.04), and COPD, a condition which is associated with an increased risk of right ventricular dysfunction, pulmonary hypertension and arrhythmias (ICD 490-496, 500-505, 506.4, J440, J441, J449).

Data collected from the QIP patients was compared to data from a group of historical control patients to assess pre-post group differences in patient outcomes following implementation of the QIP.

\section{Main outcome measures}

The two outcome measures used for this analysis were 30-day unplanned readmission (all-cause) and hospital LOS, calculated by subtracting admission day from discharge day.

\section{Statistical analysis}

Patient characteristics by group (QIP vs. control) were tabulated. Age was defined in years as the difference in years between date of birth and index admission date. Race was categorized into White, Black, and Other/ Unknown. Insurance was categorized as private (including 4 self-insured/other patients) or public. Patients were considered obese if they had a body mass index $\geq 30$. Chi-square test for categorical variables and student's t-test for continuous variables were used to compare the distributions of variables between the QIP and control groups. The two-sample exact Wilcoxon test was used to compare the LOS between QIP and controls. Logistic regression was used to estimate the probability of readmission adjusting for group (QIP vs. control), age in years, gender, race, insurance, obesity, acute myocardial infarction, HF, coronary artery disease, and atrial fibrillation. To compare the LOS between the two groups, generalized linear model with log link and Gamma distribution adjusting for group, age in years, gender, race, insurance, obesity, acute myocardial infarction, $\mathrm{HF}$, coronary artery disease, and atrial fibrillation was performed. All analyses were performed using SPSS 
22.0 (SPSS, Chicago, IL). A 2-tailed p level $<0.05$ was considered statistically significant.

\section{Results}

Of the 310 cardiopulmonary DRG patients, 166 (53.5\%) were QIP patients, and $144(46.5 \%)$ were control patients. Information regarding demographic characteristics is reported in Table 1. Compared to the controls, QIP patients were more likely to be female $(60.2 \%$ vs. $49.3 \%)$, White $(72.9 \%$ vs. $50.7 \%)$, and have private insurance $(54.8 \%$ vs. $24.3 \%)$. There were no statistically significant differences between the QIP and control patients in age or obesity, with most patients being older adults (66 \pm 17.4 years) and non-obese (85\%). In terms of the clinical characteristics, the QIP patients were less likely to have AMI $(6.6 \%$ vs. $12.5 \%, \mathrm{p}=0.07)$ and HF (25.3 vs. $59.7 \%$, p $=0.001)$. Diabetes and COPD distributions were similar between the two groups. Additionally, the QIP group had $23(13.9 \%)$ patients who were discharged to a skilled nursing facility (SNF) or a rehabilitation program while the control group did not have any patients discharged to these locations.

As $34.2 \%$ of the patients were also identified as having diabetes, data was collected on the type of ONS that was ordered for the patient during their hospital admission and are reported in Table 1 . In the QIP population, 30 $(18.1 \%)$ patients received diabetic-specific ONS, 105 $(63.3 \%)$ patients received general ONS, and $6(3.6 \%)$ patients received renal-specific ONS $(\mathrm{p}<0.001)$. Data regarding ONS type for the control group was missing for most patients $(68.1 \%)$ as ONS ordering guidelines and documentation standards were not followed consistently prior to the implementation of the QIP.

The 30-day readmission rates and hospital LOS for the QIP and control patients are presented in Table 2. Statistically significant reductions in readmission rates were seen in the QIP versus control group, where QIP patients had a 30 -day readmission rate of $13.9 \%$ versus $26.4 \%$ in the control group. This decrease represents a $55 \%$ reduction in the odds of being readmitted in the QIP group relative to the controls ( $\mathrm{OR}=0.45$, [CI 0.25- 0.79], $\mathrm{p}=0.006$ ). Similarly, in the adjusted logistic regression model, the QIP patients were 33\% less likely to be readmitted compared to the controls $(\mathrm{OR}=0.67$, [CI 0.341.33], $\mathrm{p}=0.24$ ) (Table 3).

LOS was significantly shorter in the QIP than in the control group, where QIP patients had a LOS of 5.4 \pm 5.7 days versus $6.8 \pm 5.7$ days for control patients, corresponding to a relative risk reduction (RR) of $20 \%$ $(R R=0.80,[0.67-0.94], p=0.0085)$ for the QIP group. However, the RR was 6\% after accounting for patient demographic and clinical characteristics $(\mathrm{RR}=0.94$, [CI 0.77-1.15], $\mathrm{p}=0.56)($ Table 4).
Table 1

Demographic and Clinical Characteristics of Study Subjects $(\mathrm{N}=310)$

\begin{tabular}{|c|c|c|c|}
\hline Characteristic & $\begin{array}{l}\text { QIP Group } \\
\mathrm{N}=166\end{array}$ & $\begin{array}{l}\text { Control Group } \\
\mathrm{N}=144\end{array}$ & $P$ value \\
\hline Sex, N $(\%)$ & & & 0.054 \\
\hline Male & $66(39.8)$ & $73(50.7)$ & \\
\hline Female & $100(60.2)$ & $71(49.3)$ & \\
\hline Age, in years, mean (SD) & $65.5(18.6)$ & $66.5(16.2)$ & 0.629 \\
\hline Race, N (\%) & & & $<0.001$ \\
\hline White & $121(72.9)$ & $73(50.7)$ & \\
\hline Black & $35(21.1)$ & $39(27.1)$ & \\
\hline Other/Unknown & $10(6.0)$ & $32(22.2)$ & \\
\hline Insurance, $\mathrm{N}(\%)$ & & & $<0.001$ \\
\hline Public & $71(42.8)$ & $109(75.7)$ & \\
\hline Private & $91(54.8)$ & $35(24.3)$ & \\
\hline Self-pay/Other & $4(2.4)$ & $0(0)$ & \\
\hline Discharge Disposition, N (\%) & & & $<0.001$ \\
\hline Home Independent & $110(66.3)$ & $55(38.2)$ & \\
\hline Home with Home Health & $33(19.9)$ & $89(61.8)$ & \\
\hline $\begin{array}{l}\text { Skilled Nursing Facility/ } \\
\text { Rehabilitation }\end{array}$ & $23(13.9)$ & $0(0)$ & \\
\hline Obese, $\mathrm{N}(\%)$ & $9(5.4)$ & $14(9.7)$ & 0.150 \\
\hline \multicolumn{4}{|l|}{ Diagnosis, $\mathrm{N}(\%)$} \\
\hline Acute Myocardial Infarction & $11(6.6)$ & $18(12.5)$ & 0.077 \\
\hline Heart Failure & $42(25.3)$ & $86(59.7)$ & $<0.001$ \\
\hline Atrial Fibrillation & $40(24.1)$ & $42(29.2)$ & 0.313 \\
\hline Coronary Artery Disease & $20(12.0)$ & $36(25.0)$ & 0.003 \\
\hline $\begin{array}{l}\text { Chronic Obstructive } \\
\text { Pulmonary Disease }\end{array}$ & $41(24.7)$ & $43(29.9)$ & 0.308 \\
\hline Diabetes & $50(30.1)$ & $56(38.9)$ & 0.105 \\
\hline Renal Insufficiency & $5(3.0)$ & $3(2.1)$ & 0.607 \\
\hline Hypertension & $63(38.0)$ & $55(38.2)$ & 0.965 \\
\hline ONS Type, $\mathrm{N}(\%)$ & & & $<0.001$ \\
\hline General & $105(63.3)$ & $23(16.0)$ & \\
\hline Diabetic-specific & $30(18.1)$ & $10(6.9)$ & \\
\hline Renal-specific & $6(3.6)$ & $13(9.0)$ & \\
\hline Data missing & $25(15.1)$ & $98(68.1)$ & \\
\hline
\end{tabular}

Abbreviations: SD, Standard Deviation; QIP, Quality Improvement Program; ONS Oral Nutritional Supplement

A sensitivity analysis was conducted excluding the 23 patients that were discharged to a SNF or a rehabilitation program in the QIP group. Similar results were observed as compared to the full cohort $(n=310)$. Statistically significant reductions in readmission rates were again seen in this QIP group versus controls where QIP patients had a 30-day readmission rate of $13.4 \%$ versus $26.4 \%$ for control patients $(\mathrm{p}=0.009)$ and the adjusted odds ratio was ( $\mathrm{OR}=0.68$, [CI 0.32-1.47], $\mathrm{p}=0.33$ ), which represents a $32 \% \mathrm{RR}$ in readmission rates. LOS was also significantly shorter in this QIP $(4.9 \pm 5.4$ days $)$ versus controls $(6.8 \pm 5.7)$, representing a RR reduction of $29 \%$ 
Table 2

Univariate Comparisons of 30-day Readmission Rate and Length Of Stay

\begin{tabular}{|c|c|c|c|c|}
\hline Characteristic & QIP Group N=166 & Control Group N=144 & Estimate & P value \\
\hline Readmitted within 30 days, N (\%) & $23(13.9)$ & $38(26.4)$ & $\mathrm{OR}=0.45(\mathrm{CI} 0.25-0.79)$ & 0.006 \\
\hline Length of Stay, in days, mean $( \pm$ SD) & $5.4( \pm 5.7)$ & $6.8( \pm 5.7)$ & $\mathrm{RR}=0.80(\mathrm{CI} 0.67-0.94)$ & 0.009 \\
\hline
\end{tabular}

Abbreviations: N, Number; OR, Odd Ratio; CI, Confidence Intervals; SD, Standard Deviation; QIP, Quality Improvement Program; RR, Relative Risk

Table 3

Adjusted Logistic Regression for the Probability of 30-day Readmission Rate

\begin{tabular}{|c|c|c|c|c|c|}
\hline & $\mathbf{N}$ & Odds Ratio & LCL & UCL & P-value \\
\hline Group & & & & & 0.24 \\
\hline QIP & 166 & 0.67 & 0.34 & 1.33 & \\
\hline Control & 144 & Ref & Ref & Ref & \\
\hline Gender & & & & & 0.92 \\
\hline Females & 171 & 1.02 & 0.55 & 1.87 & \\
\hline Males & 139 & Ref & Ref & Ref & \\
\hline Age & & 0.99 & 0.97 & 1.01 & 0.36 \\
\hline Race & & & & & 0.89 \\
\hline White & 184 & & & & \\
\hline Black & 74 & 1.05 & 0.51 & 2.18 & \\
\hline Other & 42 & 1.08 & 0.44 & 2.63 & \\
\hline Insurance & & & & & 0.03 \\
\hline Private & 126 & 0.48 & 0.24 & 0.95 & \\
\hline Other & 184 & Ref & Ref & Ref & \\
\hline Obesity & & & & & 0.86 \\
\hline Yes & 23 & 1.10 & 0.37 & 3.32 & \\
\hline No & 287 & Ref & Ref & Ref & \\
\hline Acute Myocardial Infarction & & & & & 0.07 \\
\hline Yes & 29 & 0.28 & 0.07 & 1.09 & \\
\hline No & 281 & Ref & Ref & Ref & \\
\hline Congestive Heart Failure & & & & & 0.24 \\
\hline Yes & 128 & 1.47 & 0.77 & 2.79 & \\
\hline No & 182 & Ref & Ref & Ref & \\
\hline Coronary Artery Disease & & & & & 0.06 \\
\hline Yes & 56 & 2.34 & 0.95 & 5.86 & \\
\hline No & 254 & Ref & Ref & Ref & \\
\hline Atrial Fibrillation & & & & & 0.89 \\
\hline Yes & 82 & 0.95 & 0.46 & 1.96 & \\
\hline No & 228 & Ref & Ref & Ref & \\
\hline
\end{tabular}

Abbreviations: N, Number; LCL, Lower Confidence Limit; UCL, Upper Confidence Limit 
Table 4

Generalized Linear Model with Gamma Distribution and Log Link to Estimate the Average Length of Stay

\begin{tabular}{|c|c|c|c|c|c|}
\hline & $\mathbf{N}$ & RR & LCL & UCL & P-value \\
\hline \multicolumn{6}{|l|}{ Group } \\
\hline QIP & 166 & 0.943 & 0.775 & 1.147 & 0.556 \\
\hline Control & 144 & Ref & Ref & Ref & \\
\hline Gender & & & & & 0.001 \\
\hline Females & 171 & 0.758 & 0.643 & 0.895 & \\
\hline Males & 139 & & Ref & Ref & \\
\hline Age & & 0.991 & 0.985 & 0.996 & 0.001 \\
\hline \multicolumn{6}{|l|}{ Race } \\
\hline White & 184 & & & & \\
\hline Black & 74 & 1.055 & 0.867 & 1.283 & 0.592 \\
\hline Other & 42 & 1.272 & 0.998 & 1.621 & 0.052 \\
\hline Insurance & & & & & 0.259 \\
\hline Private & 126 & 1.103 & 0.931 & 1.306 & \\
\hline Other & 184 & & Ref & Ref & \\
\hline Obesity & & & & & 0.134 \\
\hline Yes & 23 & 1.255 & 0.933 & 1.689 & \\
\hline No & 287 & & Ref & Ref & \\
\hline Acute Myocardial Infarction & & & & & 0.006 \\
\hline Yes & 29 & 1.659 & 1.153 & 2.388 & \\
\hline No & 281 & & Ref & Ref & \\
\hline Congestive Heart Failure & & & & & 0.081 \\
\hline Yes & 128 & 1.164 & 0.982 & 1.379 & \\
\hline No & 182 & & Ref & Ref & \\
\hline Coronary Artery Disease & & & & & 0.125 \\
\hline Yes & 56 & 0.801 & 0.604 & 1.064 & \\
\hline No & 254 & & Ref & Ref & \\
\hline Atrial Fibrillation & & & & & 0.124 \\
\hline Yes & 82 & 1.161 & 0.960 & 1.403 & \\
\hline No & 228 & & Ref & Ref & \\
\hline \multicolumn{6}{|l|}{ Discharge Disposition } \\
\hline Home & 165 & & Ref & Ref & \\
\hline Home with Home Health & 122 & 1.393 & 1.164 & 1.667 & 0.000 \\
\hline SNF/Rehab & 23 & 2.010 & 1.453 & 2.782 & $<.0001$ \\
\hline
\end{tabular}

Abbreviations: N, Number; RR, Relative Risk; LCL, Lower Confidence Limit; UCL, Upper Confidence Limit

$(R R=0.71,[0.60-0.85], p=0.0001)$. However, the RR was $6 \%$ after accounting for patient demographic and clinical characteristics $(\mathrm{RR}=0.94$, [CI 0.77-1.14], $\mathrm{p}=0.53)$ (data not tabulated).

\section{DISCUSSION}

Malnourished hospitalized cardiopulmonary patients participating in a nutrition-focused QIP experienced improved readmission rates and LOS compared to historical control patients. Interventions that result in improved outcomes for cardiopulmonary patients are noteworthy, as this population continues to have unacceptably high readmission rates (1-4, 6). Considering the high prevalence of HF alone, even relatively modest effects of implemented interventions could improve transitions in care for over 850,000 patients per year, reduce the readmission penalty for older patients of any individual hospital $\{12\}$, and therefore result in significant cost savings for patients and healthcare systems.

Malnourished hospitalized patients with HF tend to 
have a poorer prognosis than those with an adequate nutritional status, particularly patients 65 years and older who possess a multitude of nutrition risk factors due to the physiological changes of ageing (10). Malnutrition can act as a mediator of disease progression and a predictor of poor prognosis among HF patients, especially for patients in advanced disease stages (21). Nutritional intervention in HF patients however can reduce the risk of death from any cause in addition to reducing the risk of readmission for worsening HF (22). It is therefore important to provide proper nutrition care to patients with cardiopulmonary diseases, and especially older adults with HF to further optimize their health outcomes and aging process.

Prior research has demonstrated that ONS use was associated with reductions in probability of 30-day readmissions by $12.0 \%$ in acute $\mathrm{MI}$, and $10.1 \%$ in $\mathrm{HF}$. LOS decreases of $10.9 \%$ in acute MI and $14.2 \%$ in HF were associated with ONS use, as were decreases in hospitalization costs for acute MI and HF of $5.1 \%$ and $7.8 \%$, respectively. $\{19\}$ Another study investigating the impact of ONS administration on Medicare COPD patients found a $13.1 \%$ decrease in the probability of 30 -day readmissions, a $21.5 \%$ decrease in LOS, and a $12.5 \%$ hospitalization cost reduction (23). Although ONS use has been found to be associated with improved health and economic outcomes among Medicare patients with a cardiopulmonary diagnosis, ONS alone may not be adequate to reduce readmission rates and/or LOS for all patient groups, especially those with HF. To further optimize nutrition care for adult patients receiving care for cardiopulmonary conditions, similar nutritionfocused QIPs should be further researched together with other ways to improve care that can lead to reduced readmissions, and in particular reduced LOS in this population $(23,24)$.

This study revealed that a significant portion of the cardiopulmonary patients also had diabetes, thus supporting the use of diagnosis-specific ONS for the QIP patients. Diabetes adds to the complexity of care, and treating physicians need to be aware of the implications of this common comorbid condition. Specifically, clinicians should consider recommending diabetesspecific ONS as part of a comprehensive treatment plan $(25,26)$.

\section{Study limitations}

There are several limitations to this study. The data were collected from the study by Sriram et al. and thus will have similar limitations as documented previously. \{18\} ONS consumption was not recorded due to staffing and time limits. However, improved outcomes were noted even when assuming that not all patients were compliant with the ordered ONS regimen. Measures to track and increase consumption compliance would likely lead to similar, if not greater, reductions in readmissions and LOS (27). Although our study utilized QIP methodologies that can be subject to bias, QIPs also offer insight into real-world clinical experiences and provide important information about the effectiveness of nutrition programs in clinical practice settings (28). Specific to this study, some differences between the QIP and control groups were noted; a portion of patients in the QIP group were discharged to a SNF or rehabilitation facility. The fact that even these sicker patients experienced fewer readmissions and shorter LOS lends further support to the importance of nutritional therapy in patients with cardiopulmonary diseases. To mitigate the impact of the unbalanced characteristics between the QIP and historical controls, multivariate models were fit. However, it is noteworthy to mention that the study was not powered to detect a statistical difference between the two groups. To detect a statistically significant odds ratio of 0.67 with $80 \%$ power, and a 5\% significance level, a sample of 1,133 patients equally distributed between QIP and controls would be needed. Furthermore, a sensitivity analysis performed after removal of the patients that were discharged to SNF or rehabilitation facilities showed that the differences between groups in terms of readmissions and LOS remained significant. The sample size of the study also impacted the ability to subdivide this population into specific cardiopulmonary diagnoses groups. A topic for future study could be looking at how these results translate to the three diagnoses specifically - HF, COPD, or MI. As a post-hoc analysis, the outcomes may not be directly attributed to the QIP program; however, at the time of QIP implementation, no other efforts were undertaken in patients at risk for malnutrition (18). Lastly, data on ONS use was missing for a large portion of the historical control patients. This is to be expected as the QIP focused on improving ONS ordering and documentation practices.

This study highlights the importance of real-world evidence methodologies and the potential of QIPs to significantly improve key health outcomes such as 30-day readmissions and LOS among patients with cardiopulmonary diagnoses. However, additional education, awareness, and training on the benefits of nutrition care is needed among the cardiologists and pulmonologists that treat these patients as well as geriatricians to promote better outcomes and healthy aging throughout the different patient populations with HF and especially the older patients. This is particularly important given that the current treatment guidelines do not reflect the importance of nutrition in the cardiopulmonary space. For example, the HF guidelines currently view nutritional supplements as Class III-no benefit (29). However, the results of this study clearly demonstrate a benefit of a comprehensive QIP on hospitalized malnourished cardiopulmonary patients in reducing readmissions and LOS, which could in turn result in significant cost savings. Treatment of malnutrition should be evaluated in the future with QIPlike studies with larger sample sizes and prospective controlled trials (30-35), which may alter the current 
recommendations, at least in hospitalized malnourished cardiopulmonary patients. In the meantime, these results, which emphasize the importance of nutrition-focused interventions becoming a key part of the comprehensive treatment plan for cardiopulmonary patients by different healthcare providers, should be carefully considered. A conventional, disease-specific perspective may not always lead clinicians to the underlying cause of malnutrition (36). Therefore, nutrition screening/assessment and treatment should be a routine part of care for all cardiopulmonary patients across different settings of care.

Funding: Financial support for the study was provided by Abbott, Chicago, IL, USA. The ClinicalTrials.gov Identifier for this study is: NCT02262429.

Conflicts of interest: Advocate Health Care received funding from Abbott for this research. Drs. Costanzo and Brown have not received any direct funding from Abbott. Dr. Sriram has received consultancy fees from Abbott outside of the present work. Dr. Sulo and Dr. Partridge are employees of Abbott. The other authors have no conflicts of interest to report.

Ethical standard: This study was conducted according to the guidelines laid down in the Declaration of Helsinki and all procedures involving human subjects were approved by the Advocate Health Care Institutional Review Board. Verbal informed consent was obtained from all subjects. Verbal consent was witnessed and formally recorded.

\section{References}

1. Hernandez AF, Greiner MA, Fonarow GC, et al. Relationship between early physician follow-up and 30-day readmission among Medicare beneficiaries hospitalized for heart failure. JAMA. 2010;303:1716-1722.

2. Bueno H, Ross JS, Wang Y, et al. Trends in length of stay and short term outcomes among Medicare patients hospitalized for heart failure, 1993-2006. JAMA. 2010;303:2141-2147.

3. Jencks SF, Williams MV, Coleman EA. Rehospitalizations among patients in the Medicare fee-for-service program. N Engl J Med. 2009;360:1418-1428.

4. Simmering JE, Polgreen LA, Comellas AP, Cavanaugh JE, Polgreen PM Identifying patients with COPD at high risk of readmission. Chronic Obstr Pulm Dis. 2016;3(4):729-738.doi:http: / / doi.org/10.15326/jcopdf.3.4.2016.0136

5. Chen J, Normand SL, Wang Y, Krumholz HM. National and regional trends in heart failure hospitalization and mortality rates for Medicare beneficiaries, 1998-2008. JAMA. 2011;306:1669-1678.

6. Dunlay SM, Weston SA, Killian JM, Bell MR, Jaffe AS, Roger VL. Thirty Day Hospital Readmissions Following Acute Myocardial Infarction: A Community Study. Ann Intern Med. 2012;157(1):11-18. doi:10.7326/00034819-157-1-201207030-00004

7. Weiss AJ, Elixhauser A. Overview of Hospital Stays in the United States, 2012. HCUP Statistical Brief \#180. 2014; Agency for Healthcare Research and Quality, Rockville, MD. http://www.hcup-us.ahrq.gov/reports/statbriefs / sb180-Hospitalizations-United-States2012.pdf

8. Barker L, Gout S, Crowe, T. Hospital Malnutrition: Prevalence, Identification and Impact on Patients and the Healthcare System. Int J Environ Res Public Health. 2011;8(2):514-527.

9. Statistics on Hospital Stays and Hospital Readmission using 2013 National Statistics, Healthcare Cost and Utilization Project (HCUP), Agency for Healthcare Research and Quality, Rockville, MD.

10. Sheean P, Farrar IC, Sulo S, Partridge J, Schiffer L, Fitzgibbon M. Nutrition risk among an ethnically diverse sample of community-dwelling older adults. Public Hlth Nutr. 2019;22(5):894-902. doi:10.1017/S1368980018002902.

11. McKeever L, Farrar IC, Sulo S, Partridge J, Sheean P, Fitzgibbon M. Nutritional adequacy and oral nutritional supplementation in older community-dwelling adults. J Aging Res Clin Pract. 2019;8:7-14.
12. Bradley EH, Curry L, Horwitz LI, et al. Hospital strategies associated with 30-day readmission rates for patients with heart failure. Circulation: Cardiovasc Qual Outc. 2013;6:444-450.https://doi.org/10.1161/ CIRCOUTCOMES.111.000101.

13. Doehner W, Frenneaux M, Anker SD. Metabolic Impairment in Heart Failure The Myocardial and Systemic Perspective. JACC. 2014;64(13):1389-1400.

14. Roller RE, Eglseer D, Eisenberger A, Wirnsberger GH. The Graz Malnutrition Screening (GMS): a new hospital screening tool for malnutrition. Br J Nutr. 2016;115(4):650-7.

15. Snider JT, Linthicum MT, Wu Y, et al. Economic burden of community-based disease-associated malnutrition in the United States. JPEN. 2014;38(2):77S85 S.

16. Nuckols TK, Keeler E, Morton S, et al. Economic Evaluation of Quality Improvement Interventions Designed to Prevent Hospital Readmission: A Systematic Review and Meta-analysis. JAMA Intern Med. 2017;177(7):975985.doi:10.1001/jamainternmed.2017.1136

17. Leon BM, Maddox TM. Diabetes and cardiovascular disease: Epidemiology, biological mechanisms, treatment recommendations and future research. World J Diabetes. 2015;6(13):1246-1258.

18. Sriram K, Sulo S, VanDerBosch G, et al. A comprehensive nutrition-focused quality improvement program reduces 30-day readmissions and length of stay in hospitalized patients. J Parenter Enteral Nutr. 2017;41(3):384-391.

19. Lakdawalla DN, Snider JT, Perlroth DJ, et al. Can Oral Nutritional Supplements Improve Medicare Patient Outcomes in the Hospital? Forum for Health Economics \& Policy. 2014;17(2):131-151.doi:10.1515/ fhep-2014-0011

20. Ferguson M, Capra S, Bauer J, Banks M. Development of a valid and reliable malnutrition screening tool for adult acute hospital patients. Nutrition. 1999;15(6):458-64.

21. Agra Bermejo RM, González Ferreiro R, Varela Román A, et al. Nutritional status is related to heart failure severity and hospital readmissions in acute heart failure. Int J Cardiol. 2017;230:108-114.doi:10.1016/j.ijcard.2016.12.067

22. Bonilla-Palomas JL, Gámez-López AL, Castillo-Domínguez JC, et al. Nutritional Intervention in Malnourished Hospitalized Patients with Heart Failure. Arch Med Res. 2016;47(7):535-540.doi:10.1016/j.arcmed.2016.11.005

23. Thornton Snider J, Jena AB, Linthicum MT, et al. Effect of Hospital Use of Oral Nutrition Supplementation on Length of Stay, Hospital Cost, and 30-Day Readmissions Among Medicare Patients With COPD. CHEST. 2015;147(6):1477-1484.

24. Babb EB, Rohrer J. Oral nutritional supplement use in relation to length of stay in heart failure patients at a regional medical center. J Eval Clin Pract. In press.doi:10.1111/jep.12762

25. Whitman D. Nutrition management of diabetes in acute care. Can J Diabetes. 2014;38(2):90-3.doi:10.1016/j.jcjd.2014.01.00

26. Swift CS, Boucher JL. Nutrition therapy for the hospitalized patient with diabetes. Endocr Pract. 2006;12(3):61-7.

27. Sriram K, Sulo S, Summerfelt WT. Author Response. J Parenter Enteral Nutr. 2017;41(4):528-529.

28. Drazen JM, Harrington DP, McMurray JJV, Ware JH, Woodcock J. Evidence for health decision making - Beyond randomized, clinical trials. NEJM. 2017;377(5):465-475.

29. Yancy CW, Jessup M, Bozkurt B, et al. 2013 ACCF/AHA guideline for the management of heart failure: a report of the American College of Cardiology Foundation/American Heart Association Task Force on Practice Guidelines. 2013;62(16):e147-239.

30. Azuma J, Sawamura A, Awata N. Usefulness of taurine in chronic congestive heart failure and its prospective application. Jpn Circ J. 1992;56:95-9.

31. Fazio S, Sabatini D, Capaldo B, et al. A preliminary study of growth hormone in the treatment of dilated cardiomyopathy. N Engl J Med. 1996; 334:809-14.

32. Ferrari R, De Giuli F. The propionyl-L-carnitine hypothesis: an alternative approach to treating heart failure. J Card Fail. 1997;3:217-24.

33. Ghatak A, Brar MJ, Agarwal A, et al. Oxy free radical system in heart failure and therapeutic role of oral vitamin E. Int J Cardiol. 1996;57:119-27.

34. Hamilton MA, Stevenson LW. Thyroid hormone abnormalities in heart failure: possibilities for therapy. Thyroid. 1996;6:527-9.

35. Soja AM, Mortensen SA. Treatment of congestive heart failure with coenzyme Q10 illuminated by meta-analyses of clinical trials. MolAspects Med. 1997;18:159-68.

36. Evans C. Malnutrition in the elderly: a multifactorial failure to thrive. Perm J. 2005; 9(3): 38-41. 